\title{
A Model to Evaluate the Safeness of My City Luonan Qiu
}

\author{
School of North China Electric Power University, Beijing 102206,China;
}

532039615@qq.com

\begin{abstract}
Keywords: reaction index, reaction index, objective weighting method, objective weighting method, objective weighting method
\end{abstract}

\begin{abstract}
We develop a model to analyze the data and then create a safety rating to specify a measure of how safe My City is. The goal is to use safety rating so as to evaluate the safeness of My City. Using historical data from the police reports in My City, we capture the essential elements of urban public safety, like crime rate, crime severity and detection rate. To establish a social security evaluating indicator system, we entrusts with the weight to each of elements by means of Expert's Judgement. Next, we use our full model based on each weight to evaluate the safeness of My City. Our suggested solution, which is easy to implement, includes a social security evaluating indicator system and a safety rating of the city.
\end{abstract}

\section{Introduction}

Introduction Urban public safety has become a primary concern of urban sustainability in recent years due to escalated potential loss if urban public safety is severely breached. So it is necessary to build a social security evaluating indicator system by analyzing the raw data.

Focusing on city crime and safety, based on AHP(analytic hierarchy process), we develop a mathematical model for safety rating for My City. First, according to the () we divide all different types of crime into three areas, which are disrupting public order crime, violent crimes, property crimes, and give weights to each of them. Next, we entrusts with the weight to each of types of the crime included in different areas to create the individual crime evaluation index. The weight of each crime is established by considering the crime rate, crime severity and detection rate. Finally by using FAHP(fuzzy analytic hierarchy process) to calculate hierarchical single sort (weight) and total ordering to obtain the social security evaluating indicator system. Combining qualitative method with quantitative method organically, all the objectives, principles which are difficult to quantify are translated into a multi-level single objective decision-making problem. Through calculating the hierarchical relationship, the results are easy for policy makers to understand and grasp.

\section{Background}

Security and stability of a city is the basic guarantee of the city happiness. In order to create a good social security environment to ensure the safety of people's lives and property, effectively combatting criminal Activities is very important.

Currently, the social security situation in the world still do not have a unified evaluation index system. The number of criminal cases is regarded as the main indicator to evaluate the city safety by many countries or regions.

Such as Japan regards the number of the cases according to the police reports as the main indicator. However, Taiwan based primarily on the increase of the violence and theft cases. Obviously, it is not comprehensive to assess the social security situation by considering only a few aspects. since the 1970s, relevant organizations of United Nations has carried out Four global crime surveys.Comparing different degree of the crime harm from different countries, the organizations use the statics as the city safe evolution criteria.At present, the evaluation methods are summarized as follows:

Individual crime statistics: Using some representative Crime statistics index to reflect social security and using the index as the basis to compare security situation of different countries. 
Multiple objective statistic indicators: Comparing features of several representative cases to evaluate the security situation.

Combining subjective indicators with objective indicators: In addition to the traditional indicators, such as the crime rate, Detection rate Etc.

The method also combines subjective indicators like public evaluation of the priorities of the police, and so trying to show the whole Availability Security System.

Our assessment of the city safety will apply the three method above, which is easy to implement, includes a social security evaluating indicator system and a safety rating of the city.

\section{Assumption}

- In this model we assume that judgments for seriousness of cases from part of public whom have been investigated can stand for all of the publics'judgments.

- We assume that the cases' Occurrence frequency are same in the following days which are without an investigations.

- We ignore the influence from those cases which haven’t happened in investigation Dates.

- We ignore the influence from factor of locales.

- The police resources is spreader symmetrical in this area.

- The density of population is a constant.

- The non-native population density is a constant.

\section{Models}

We define some terms and phrases: reaction index: Criminal cases which have a direct impact on urban safety are regarded as the reaction index, and these 16 types of crime are divided into three areas : the violent crimes, the property crimes and the disturbing public crime.

Rreaction index: The crime rate is greatly influenced by the rate of the non-local population, therefore the rate of the non-local people is regarded as the reference index.

Objective weighting method: AHP(analytic hierarchy process)

Subjective weighting method: Expert valuation method.

Objective weighting method: Individual crime evaluation index means that the harm of different types of crime to the city. The larger the weight, the greater the harm to the safety of the city.

Objective weighting method: There are several methods of calculating the comprehensive index, like the Superposition method, the mathematics average method, the weighted average method, the quadratic sum method, and the RMS method. We adopt the weighted average method in our model.

\section{Summary}

First, according to AHP(analytic hierarchy process), We set up assessment indicators used in the urban safety assessment model. Because criminal cases have a direct impact on urban safety, the types of crime are regarded as the reaction index, and these 16 types of crime are divided into three areas called crime classification criterion on the basis of the violent crimes, property crimes and disturbing public crime. The crime rate is greatly influenced by the rate of the non-local people, therefore the rate of the non-local people is regarded as the reference index. Finally we can obtain the hierarchy of the model . Next we refine the data and materials provided by the attachment, and then classify different types of crimes according to the crime classification criterion. After calculating the crime rate, detection rate, daily crime rate, we get the Table $\mathbf{1}$ 
Table 1: occurrences of the three main types of crime cases

\begin{tabular}{lcc}
\hline Type & The total number of cases & The average daily number of cases \\
Disturbing public crime & 748 & 53 \\
Property crime & 4776 & 341 \\
Violent crime & 5208 & 372 \\
\hline \hline
\end{tabular}

We adopt the weighted average method in our model to calculate the comprehensive index. As for the individual crime evaluation index, in order to prevent the result of the importance of weight degree is not conform to the practical situation by using single subjective weighting method. we can obtain the order of the importance of weight, we can use the objective weighting method and subjective weighting method separately, and then compare each method's result. However this method is time-wasting and need a large number of calculation because we need to adjust the method until the two results are the same. Therefore we adopt the method combining analytic hierarchy process and expert valuation method to determine the weight.

First determine the order of index by using the expert valuation method, and then obtains the judgment matrix of AHP method and calculate the weight of each index. Getting higher accuracy of the index weight in the lower cost and shorter time, which can largely eliminate the random of the subjective weighting method. we have to consider the crime security, detection rate, and crime rate when using the expert valuation method ordering the individual crime evaluation index. This is because if the case is classified by their nature. for single case, the nature more abominable, the greater impact on society. but for the crime rate, for a single case, the higher the frequency, the greater the impact on society. For example, murder is much more harmful than the theft on our society, however the number of the occurrence of murder is much smaller than the theft, so the social harmfulness of theft may higher than murder. From this, we can get the ranking of the severity of each individual.

\section{References}

[1] Yike Ou,Zhixing Zuo,Application Research of urban community public safety evaluation based on Analytic Hierarchy Process,Central South University,2011

[2] Yongdong Zhou,Zongzhi Wu,Research on evaluation index and method of safe community,Capital University of Economics and Business,2005 